\title{
The Clinical Relevance of Frequent Germline Genetic Variants Detected by Targeted Sequencing in Patients With Rectal Adenocarcinoma (READ)
}

\author{
KEVIN CHIH-YANG HUANG ${ }^{1,2 \#}$, SHU-FEN CHIANG ${ }^{3,4 \#}$, TAO-WEI KE ${ }^{5}$, \\ WILLIAM TZU-LIANG CHEN ${ }^{5}$, TSUNG-WEI CHEN ${ }^{6,7 *}$ and KUN-SAN CLIFFORD CHAO ${ }^{3 *}$ \\ ${ }^{1}$ Translation Research Core, China Medical University Hospital, \\ China Medical University, Taichung, Taiwan, R.O.C.; \\ ${ }^{2}$ Department of Nutrition, HungKuang University, Taichung, Taiwan, R.O.C.; \\ ${ }^{3}$ Cancer Center, China Medical University Hospital, China Medical University, Taichung, Taiwan, R.O.C.; \\ ${ }^{4}$ Lab of Precision Medicine, Feng-Yuan Hospital, Ministry of Health and Welfare, Taichung, Taiwan, R.O.C.; \\ ${ }^{5}$ Department of Colorectal Surgery, China Medical University Hospital, \\ China Medical University, Taichung, Taiwan, R.O.C.; \\ ${ }^{6}$ Department of Pathology, China Medical University Hospital, \\ China Medical University, Taichung, Taiwan, R.O.C.; \\ ${ }^{7}$ Graduate Institute of Biomedical Science, China Medical University, Taichung, Taiwan, R.O.C.
}

\begin{abstract}
Background: The progression of colorectal cancer (CRC) mainly stems from the occurrence of somatic mutation. However, there is little information that can be used to comprehensively analyse the importance of germline variants in CRC patients. Patients and Methods: The candidate germline variants between tumor relapse and cured rectal adenocarcinoma (READ) were firstly filtered by whole-exome sequencing $(n=4)$, and validated by targeted sequencing and associated with clinical outcome in READ $(n=48)$. Results: We identified 9 pathogenic germline variants that were clinically associated with survival outcome in READ, including TIPIN, TLR1, TLR10, OR4D6, IGSF3, UBBP4, OR6J1, FAM208A and DISC1. Patients carrying these germline susceptibility variants had an increased risk of poor survival outcome compared to those without these variants. Conclusion: Not only the tumor
\end{abstract}

This article is freely accessible online.

\footnotetext{
\#These authors contributed equally to this study.

*These authors contributed equally to this study.

Correspondence to: K. S. Clifford Chao, Cancer Center, China Medical University Hospital, China Medical University, Taichung, Taiwan, 40402, R.O.C. Tel.: +886 422052121 ext. 2976, Fax: +886 422075011, e-mail: d94032@mail.cmuh.org.tw
}

Key Words: Targeted sequence panel, whole exome sequence, rectal adenocarcinoma, personalized medicine. genome, but also the germline sequence must be analysed to depict the overall genetic profile, providing potential therapeutic strategies for personalized medicine.

Colorectal cancer (CRC) is the leading malignancy of cancer-related deaths worldwide (1), and $\sim 30 \%$ of CRC cases are rectal adenocarcinomas (READ) (2). The standard therapeutic strategy for locally advanced rectal adenocarcinoma (LARC) is preoperative (neoadjuvant) chemoradiotherapy (neoCRT), which has been reported to control local disease and reduce distant metastasis (3-5). However, a diverse range of pathologic responses was achieved in rectal adenocarcinoma patients treated with neoCRT (6), suggesting that tumor heterogeneity affected by genetic and epigenetic variations may influence the therapeutic efficacy of neoCRT in patients with locally advanced rectal adenocarcinoma (7).

Cancer susceptibility genes affect cancer formation via different signalling pathways that impact the responses to treatments and the immunosurveillance of cancer cells (8). Therefore, genomic medicine has been well accepted for the diagnosis and treatment of certain types of malignancies such as chronic myeloid leukaemia (9), lung cancer (10) and colorectal cancer (11). These critical genetic mutations determine the therapeutic efficacy; therefore, detecting oncogenic mutations has become standard practice before cancer treatment. Commercial targeted sequencing cancer panels have been developed to identify somatic mutations that can be clinically actionable $(12,13)$, including mutations 
at codon $12 / 13$ of RAS (14), mutations at codon 600 of BRAF (15), mutations of PIK3CA, microsatellite instability (MSI) and others $(11,16)$. These genetic profiles significantly influence the therapeutic strategies, such as EGFR-targeted therapeutic antibody cetuximab, used for CRC patients (17). KRAS mutations adversely affect patients' responses to cetuximab modalities. Furthermore, mutations in EGFR may lead to unresponsive to cetuximab. Mutations in the downstream of EGFR signalling genes such as PIK3CA or BRAF may also adversely affect treatment response (18). Moreover, genetic polymorphisms such as UGTIAl have been reported to be associated with excess toxicity of the chemotherapeutic drug irinotecan (19), which suggests that germline mutations may contribute to cancer susceptibility. However, there is still no comprehensive analysis on the correlation between germline variants and cancer susceptibility, treatment responses and drug-related toxicity in rectal adenocarcinoma. It is important to identify the individual underlying variations that can potentially affect treatment to improve precision medicine and personalized oncology (13).

Herein, we utilized germline whole-exome sequencing (WES) to identify germline susceptibility variants, and validated these germline variants that may impact clinical outcome in rectal adenocarcinoma by targeted sequencing. Based on the results of WES, we comprehensive investigated the mutational status of 400 variants in 183 genes in a cohort of rectal adenocarcinoma cases. The results will help us understand the genetic background, providing information for clinical implementation in rectal adenocarcinoma.

\section{Patients and Methods}

Tumor specimen for whole-exome sequencing (WES). Our study involved 4 rectal adenocarcinoma patients who received tumor resection at the Department of Colorectal Cancer Surgery, China Medical University Hospital with written informed consent that approved by Institutional Research Board of China Medical University Hospital (CMUH105-REC2-072, Taichung, Taiwan, ROC). For whole exome sequencing, tumor tissues were stained with haematoxylin and eosin, and the selected tumor region was confirmed to be $>70 \%$ tumor cell population by pathologist as previously described $(20,21)$. For each tumor tissue, matched normal peritumoural tissue sample was taken as a reference.

Genomic DNA (gDNA) from tumor and matched normal peritumoural samples was extracted by QIAamp DNA Kit (Qiagen, Dusseldorf, Germany) according to the manufacturer's manual. Total DNA then was used to generate genomic DNA libraries with a custom targeted capture kit designed by the Agilent SureSelect Human All Exon V5 Kit (Agilent Technologies, Santa Clara, CA, USA) (22).

Exome sequencing was performed on gDNA from tumor and matched normal peritumoural samples. The amplified DNA libraries were sequenced and analyzed by the Illumina HiSeq X Ten Genome Analyzer (Illumina, San Diego, CA, USA), yielding $150 \mathrm{bp}$ of paired-end sequence reads.
The sequencing reads of gDNA were then aligned to the human genome (GRCh37) and subsequently realigned by the Genome Analysis Toolkit (GATK) to correct the individual genomes with respect to the reference genome. The final BAM files were used for variant calling, which carried out by a series of published software tools in the single nucleotide polymorphism database (dbSNP138). All variants in coding regions were visually inspected using the Integrative Genomics Viewer (IGV) for validation.

Polymorphism validation by targeted sequencing. Forty-eight rectal cancer patients with biopsy-proven tumors were collected at the China Medical University Hospital (CMUH) in the study cohort (23). From resected tumors and paired non-tumor tissues DNA and enriched DNA was extracted, by a custom QIAseq Targeted DNA Panel (cat\# CDHS-12889Z-347), a multiplex PCR target enrichment panel for relevant target genes. The DNA panel was designed using the Qiagen QIAseq designer website (https://www.qiagen.com/ us/shop/genes-and-pathways/custom-products/custom-arrayproducts/qiaseq-targeted-dna-panels/\#design). The PCR-amplified 400 500 bp fragments were excised in the process of gel extraction and purified using a QIAquick Gel Extraction Kit (Qiagen). No negative control PCR product existed. The prepared library was validated on an Agilent 2100 Bioanalyzer and a real-time PCR system before loading on to the up-to-date NGS sequencer, NextSeq500 (Illumina). The sequences of $2 \times 150$ bp paired-end reads were produced from the sequencer following the instructions of the manufacturer.

Statistical analysis. JMP Pro statistical software version 12 (SAS Institute, NC, USA) was used to perform the statistical analysis, including Student's $t$-test, Pearson's Chi-square test and Fisher's exact test. All statistical analysis reported a two-sided $p$-value $<0.05$ as a significance level. Cox regression analysis was utilized for univariate and multivariate models to estimate the hazard ratios (HRs) and 95\% confidence intervals (CIs) $(24,25)$. The KaplanMeier survival analysis (KM curve) was used to estimate the fiveyear OS and DFS. The survival times were defined based on the period between surgery and events such as tumor relapse and death. The univariate comparison for survival analysis was performed using the log-rank test.

\section{Results}

Potential genomic variants were identified in rectal adenocarcinoma by whole exome sequence (WES). To identify the candidate variants that could be clinically associated with tumor relapse and survival outcome, we selected rectal adenocarcinoma patients with tumor relapse within 2 years (tumor relapse group) and patients with cured cancer (reference group) after neoCRT treatment and surgery. Then, tumor and non-tumor genomic DNA was extracted from FFPE samples for WES (Figure 1A and Table I). In terms of dbSNP annotation, we compared these two groups and identified 632 genetic variants (SNVs and small indels) that were observed only in the tumor relapse group (Figure 1B). These findings highlight that these genetic variants may be associated with tumor relapse in rectal adenocarcinoma after treatment. 

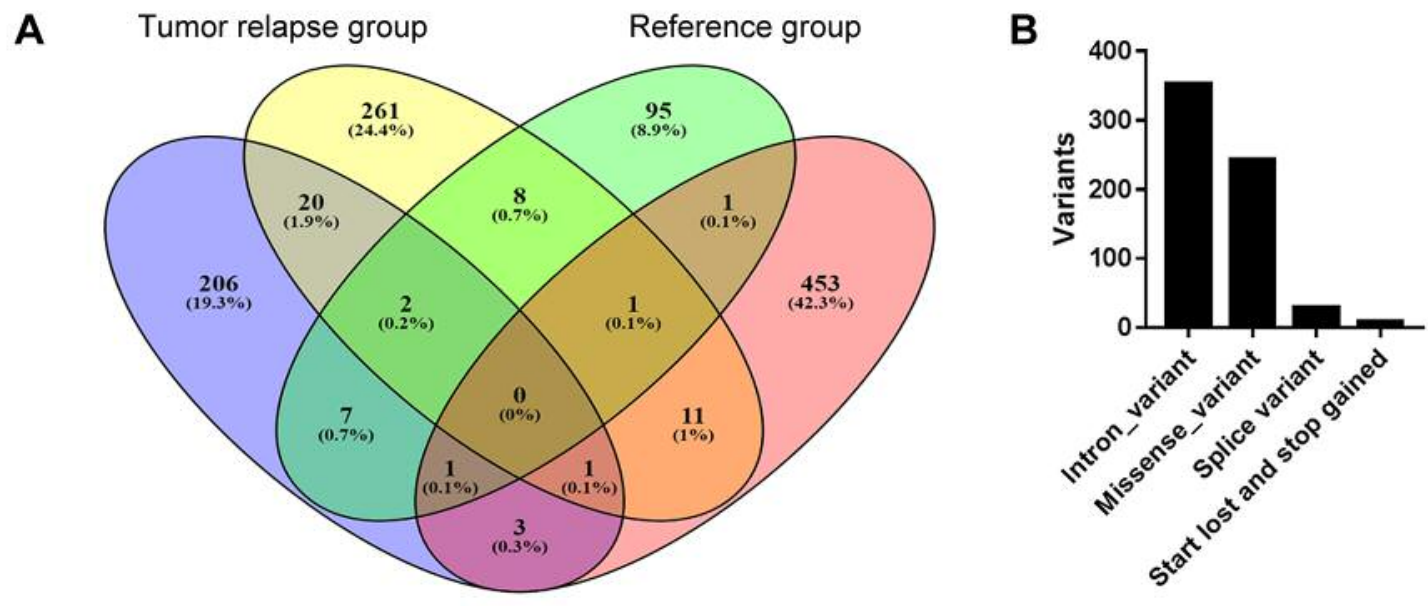

Figure 1. WES analysis identified genetic variants that were associated with tumor relapse in rectal adenocarcinoma patients. (A) Venn diagram of unique and shared genetic variants between tumor relapse (tumor relapse group) and cured patients (reference group). (B) The unique variants were identified in tumor relapse patients.

Table I. The clinicopathological characters of rectal adenocarcinoma for WES (N=4).

\begin{tabular}{|c|c|c|c|c|c|c|c|c|c|c|c|c|c|c|c|}
\hline & Gender & Age & $\begin{array}{c}\mathrm{cT} \\
\text { stage }\end{array}$ & $\begin{array}{c}\mathrm{cN} \\
\text { stage }\end{array}$ & $\begin{array}{c}\mathrm{cM} \\
\text { stage }\end{array}$ & $\begin{array}{l}\text { Clinical } \\
\text { stage } \\
\left(7^{\text {th }}\right. \\
\text { AJCC })\end{array}$ & $\begin{array}{c}\mathrm{pT} \\
\text { stage }\end{array}$ & $\begin{array}{l}\mathrm{pN} \\
\text { stage }\end{array}$ & $\begin{array}{l}\text { Pathological } \\
\text { stage } \\
\left(7^{\text {th }}\right. \\
\text { AJCC })\end{array}$ & $\begin{array}{l}\text { Perineural } \\
\text { invasion } \\
\text { (PNI) }\end{array}$ & $\begin{array}{c}\text { Lympho- } \\
\text { vascular } \\
\text { invasion } \\
\text { (LVI) }\end{array}$ & $\begin{array}{l}\text { Local } \\
\text { recurrence } \\
\text { (LR) }\end{array}$ & $\begin{array}{l}\text { Time } \\
\text { to } \\
\text { LR } \\
\text { (days) }\end{array}$ & $\begin{array}{c}\text { Distant } \\
\text { metastasis } \\
(\mathrm{DM})\end{array}$ & $\begin{array}{c}\text { Time } \\
\text { to } \\
\text { DM } \\
\text { (days) }\end{array}$ \\
\hline Tumor relapse \#1 & Male & 53 & 3 & 0 & 0 & IIA & 2 & $2 \mathrm{~A}$ & IIIB & No & No & Yes & 80 & Yes & 616 \\
\hline Tumor relapse \#2 & Male & 81 & 3 & 0 & 0 & IIA & 3 & $1 \mathrm{C}$ & IIIB & Yes & Yes & Yes & 260 & Yes & 452 \\
\hline Cured \#1 & Female & 53 & 3 & 0 & 0 & IIA & 2 & 0 & I & No & No & No & NA & No & NA \\
\hline Cured \#2 & Female & 46 & 3 & 0 & 0 & IIA & 1 & 0 & I & No & No & No & NA & No & NA \\
\hline
\end{tabular}

Clinical characteristics and survival outcome in rectal adenocarcinoma patients. To validate that these genetic variants are associated with tumor relapse in rectal adenocarcinoma, a cancer hotspot panel, the Custom QIAseq Targeted DNA Panel, was utilized to screen 48 archival FFPE samples obtained from rectal adenocarcinoma patients (Table II). This targeted DNA panel includes the immune-related genes and genetic variants that were identified by the WES. Table II presents the clinical pathological characteristics of the rectal adenocarcinoma patients $(n=48)$. The majority of patients were female $(68.8 \%)$. Twenty-four patients $(50 \%)$ received pre-operative concurrent chemoradiotherapy, and 24 patients received post-operative concurrent chemotherapy. Lymphovascular invasion (LVI) was observed in 24 rectal cancer patients, and perineural invasion (PNI) was observed in 18 rectal cancer patients (26).

With this cancer hotspot panel, we screened 183 genes (347 primer fragments), and the variants were filtered based on coverage levels above 40x and $p$-value $<0.05$ to exclude the common variants, and these hotspot mutations were identified in 48 cases at variable frequency (Table III). Frequent somatic mutations were identified in RAS (p.G12V, p.G12S, p.G12D, p.G13D, p.A59T, p.E61H, p.K117N, p.A146T) and BRAF (p.V600E). Other germline variants included TIPIN (rs2063690, c.332C $>$ G, p.A111G), TLR1 (rs4833095, c.743A $>$ G, p.N248S), IGSF3 (rs138851517, c.1916T>C, p.I639T), OR4D6 (rs1453541, c.788T >C, p.M623T), OR6J1 (rs1753430, c.748C>T, p.P250S), SNX31 (rs2248609, c.926A>G, p.E309R), TLR10 (rs4129009, c. $2323 \mathrm{~A}>\mathrm{G}$, p.I775L), UBBP4 (rs72842072, c.5G $>\mathrm{A}$, p.R2E), DISC1 (rs3738401, c.791G >A, p.R264E), and FAM208A (rs2291498, c.4120A >G, p.I1374V).

Genetic variants were clinically associated with 5-year DFS and 5-year OS in rectal adenocarcinoma. In these 48 rectal cancer patients, the variants were identified at variable frequencies (Figure 2A and Table IV): RAS (39.6\%), TIPIN (41.7\%), TLR1 (31.2\%), IGSF3 (6.3\%), OR4D6 (35.4\%), 
Table II. Clinicopathological parameters in this study (n=48).

\begin{tabular}{|c|c|}
\hline Clinicopathological parameters & Total cases $(\%)$ \\
\hline & 48 \\
\hline \multicolumn{2}{|l|}{ Age } \\
\hline$<65$ & $30(62.5 \%)$ \\
\hline$\geq 65$ & $18(37.55)$ \\
\hline \multicolumn{2}{|l|}{ Gender } \\
\hline Female & $33(68.8 \%)$ \\
\hline Male & $15(31.2 \%)$ \\
\hline \multicolumn{2}{|l|}{ Clinical TNM stage ( $7^{\text {th }}$ AJCC) } \\
\hline I & $4(8.3 \%)$ \\
\hline II & $35(72.9 \%)$ \\
\hline III & $5(10.5 \%)$ \\
\hline IV & $4(8.3 \%)$ \\
\hline \multicolumn{2}{|l|}{ pT stage } \\
\hline $0-2$ & $4(8.3 \%)$ \\
\hline $3-4$ & $44(91.7 \%)$ \\
\hline \multicolumn{2}{|l|}{ pN stage } \\
\hline Negative & $25(52.1 \%)$ \\
\hline Positive & $23(47.9 \%)$ \\
\hline \multicolumn{2}{|l|}{ Pathologic TNM stage ( $7^{\text {th }}$ AJCC) } \\
\hline I & $3(6.3 \%)$ \\
\hline II & $22(45.8 \%)$ \\
\hline III & $20(41.6 \%)$ \\
\hline IV & $3(6.3 \%)$ \\
\hline \multicolumn{2}{|l|}{ Preoperative CRT } \\
\hline Yes & $24(50 \%)$ \\
\hline No & $24(50 \%)$ \\
\hline \multicolumn{2}{|l|}{ Histological grade } \\
\hline Well & $2(4.1 \%)$ \\
\hline Moderate & $44(91.8 \%)$ \\
\hline Poor & $2(4.1 \%)$ \\
\hline \multicolumn{2}{|l|}{ LVI } \\
\hline Absent & $24(50 \%)$ \\
\hline Present & $24(50 \%)$ \\
\hline \multicolumn{2}{|l|}{ PNI } \\
\hline Absent & $30(62.5 \%)$ \\
\hline Present & $18(37.5 \%)$ \\
\hline
\end{tabular}

LVI: Lymphovascular invasion; PNI: perineural invasion.

OR6J1 (10.4\%), SNX31 (12.5\%), TLR10 (33.3\%), UBBp4 (4.2\%), DISC1 (35.4\%) and FAM208A (6.3\%). In terms of disease-free survival (DFS) and overall survival (OS), rectal adenocarcinoma patients with tumors harbouring RAS somatic mutations had shorter DFS (Kaplan-Meier log-rank test, $63.8 \% v s .39 .4 \%, p=0.0533)$ and poorer OS $(74.5 \% v s$. $45 \%, p=0.0111)$ than patients without these mutations. Moreover, multiple somatic mutations in RAS, BRAF and PIK3CA were associated with worse 5-year DFS $(67.9 \%$ vs. $38.4 \%, p=0.0308$, Figure 2B) and 5-year OS (79.8\% vs. $43.6 \%, p=0.0028$ ) of patients.

Similarly, patients with germline mutations had shorter DFS and OS than patients without mutations. IGSF3-I639T variants were associated with poorer 5-year DFS $(58.5 \% \mathrm{vs}$. $0.0 \%, p<0.0001$, Figure 2C) and 5-year OS (68.1\% vs. $1.3 \%$,
Table III. Variants detected by the cancer hotspot panel in rectal adenocarcinoma.

\begin{tabular}{ll}
\hline Gene & Mutations detected \\
\hline KRAS & p.Gly12Val, p.Gly12Ser, p.Gly12Asp, p.Gly13Asp, \\
& p.A59T, p.Gln61His, p.K117N, p.Ala146Thr \\
NRAS & p.Gly12Val, p.Gly12Ser, p.Gly12Asp, p.Gly13Asp, \\
& p.A59T, p.Gln61His, p.K117N, p.Ala146Thr \\
PIK3CA & p.Glu542Lys, p.Glu545Asp, p.Glu545Gly, \\
& p.Glu545Lys, p.Gln546His, p.His1047Arg \\
BRAF & p.Val600Glu \\
TIPIN & p.A111G \\
TLR1 & p.S602I, p.N248S \\
TTF1 & p.A290S \\
IGLV3-21 & p.V66I, p.D68Y \\
IGSF3 & p.I639T \\
KIR2DS4 & p.K156R \\
OR4D6 & p.M263T \\
OR6J1 & p.P250S \\
SNX31 & p.E309R \\
TLR10 & p.I775L \\
TLR3 & p.L412F \\
TLR5 & p.F616L \\
TLR8 & p.M1V \\
TMPRSS11A & p.M1I \\
UBBp4 & p.R2E \\
FPR1 & p.E346A \\
DISC1 & p.R264E \\
FAM208A & p.I374V \\
\hline &
\end{tabular}

$p<0.0001)$. TLR10-I775L variants were associated with poorer 5-year DFS (63.8\% vs. 36.3\%, $p=0.0465$, Figure 2D) and 5-year OS (77.2\% vs. 39.1\%, $p=0.0059)$. UBBp4-R2E variants were associated with poorer 5-year DFS (57.2\% vs. $1.4 \%, p=0.0044$, Figure 2E) and 5-year OS (66.9\% vs. $0.0 \%$, $p=0.0003)$. FAM208A-I1374V variants were associated with poorer 5-year DFS (58.3\% vs. 3.6\%, $p=0.0055)$ and 5-year OS $(68.0 \%$ vs. $7.5 \%, p=0.0004)$.

The variant OR6J1-P250S (58.8\% vs. 16.6\%, $p=0.0138$ ) was correlated with poor 5-year DFS. Some variants, such as TIPIN-A111G $(76.8 \%$ vs. $45.3 \%, p=0.0125)$, TLR1N248S (75.1\% vs. 40.7\%, $p=0.025)$, OR4D6-M263T $(82.4 \%$ vs. 52.9\%, $p=0.0274)$, SNX31-E309R (68.7\% vs. 27.8\%, $p=0.0366)$, and DISC1-R264E $(74.0 \%$ vs. $44.5 \%$, $p=0.0253$ ), were significantly correlated with poor 5-year OS. Taken together, these results indicate that several germline mutations were significantly associated with 5 -year survival in rectal adenocarcinoma.

Prognostic value of these variants on survival outcome of rectal adenocarcinoma patients. We then evaluated the risk factors for 5-year DFS and 5-year OS by univariate analysis. We found that patients with LVI, PNI, or variants of RAS/BRAF, IGSF3-I639, OR6J1-P250 and UBBp4-R2 had 
A

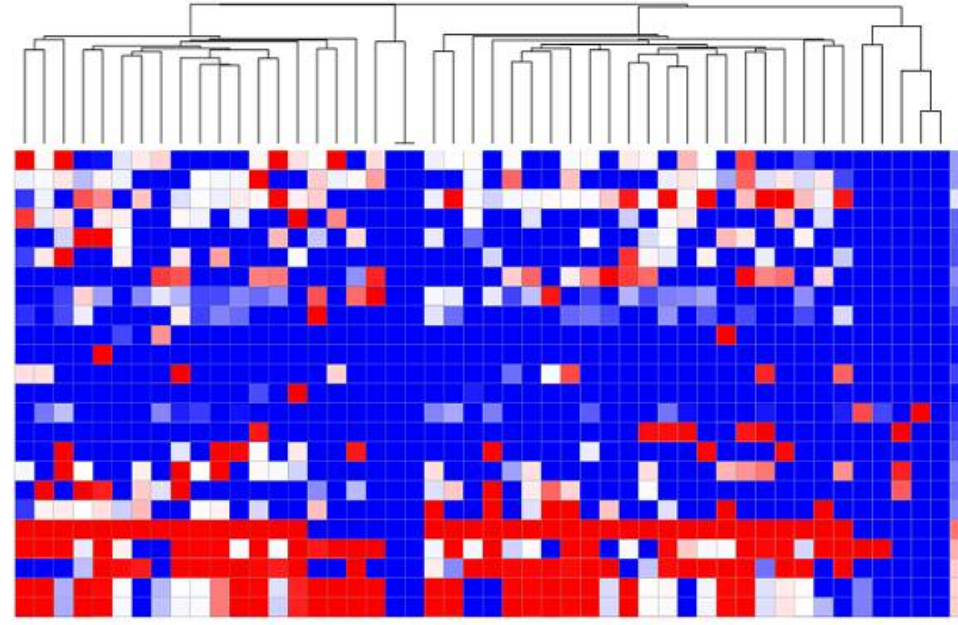

OR6I1 D.Pro250Ser TTF1. D.Ala290Ser GUF1 DL Leu58Pro DISC1_p.Arg264GIn UBBP4 DAR2GIn TLR5_p.Phe616Leu OR4D6. D. Met263Thr IGSF3_p.Ser651lle IGSF3 3 D.lle639Thr PIK3CA D. Glu545LYS IGSF3 D. Aro671Tro KRAS D.GIV13ASD KRAS D.GIY12ASD TMPRSS11A D.Met1? KIR2DS4_D.Lys156Arg FAM208A D.lle1374Val SNX31_D.Gin309Arg TIPIN D.Ala111Gly TLR1 RSer602lle TRR1 R.Glu346Ala
TLR_p.Met1? IGLV3-21_. D. Val66lle IGLV3-21 D.AsD68Ty

B

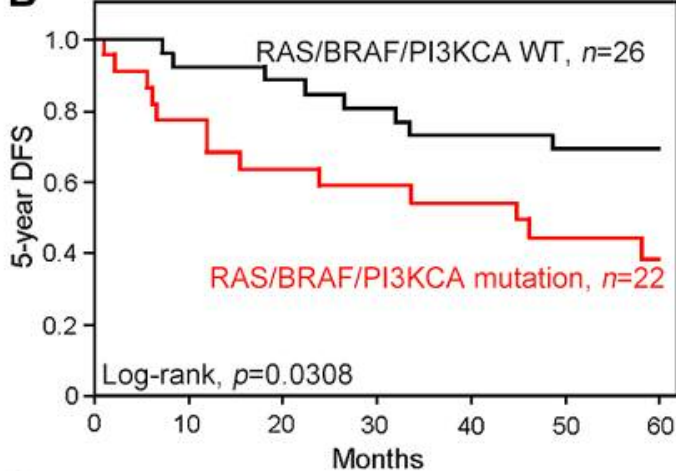

D

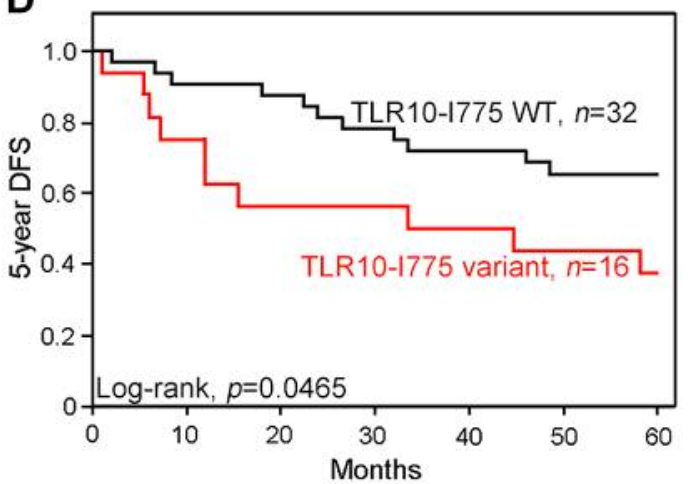

C

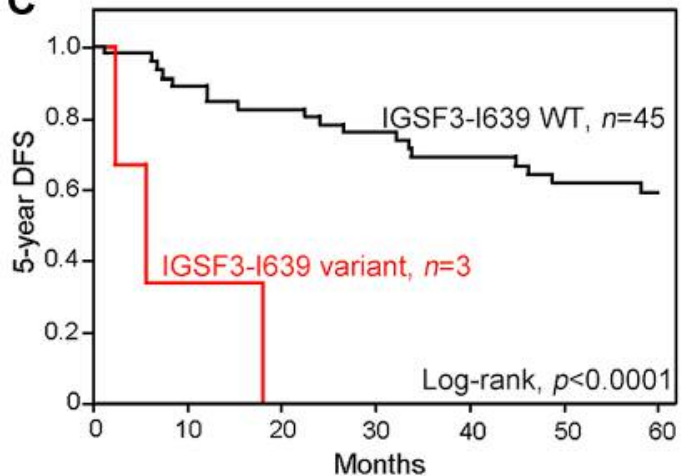

E

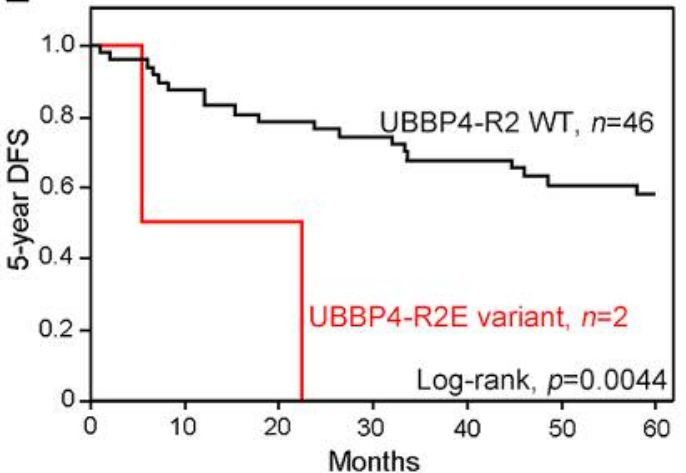

Figure 2. Targeted sequencing identidied several gemeline variants that were clinically associated with 5-year disease-free survival in rectal adenocarcinoma (n=48). (A) Heatmap of germline variants in rectal adenocarcinoma $(n=48)$. (B) Somatic mutations in RAS, BRAF and PI3KCA are clinically associated with 5-year DFS in READ ( $p=0.038, n=48)$. (C) Germline variant of IGSF3-I639 is associated with 5-year DFS in READ $(p<0.001, n=48)$. (D) Germline variant of TLR10-I775 is associated with 5-year DFS in READ $(p=0.0465, n=48)$. (E) Germline variant of UBBP4$R 2$ is associated with 5-year DFS in READ ( $p=0.0044, n=48)$.

a statistically increased risk of 5-year DFS compared with the other subgroup patients (Table V). Moreover, patients with variants of RAS/BRAF, TIPIN-A111, TLR1-N248, IGSF3- I639, OR4D6-M263, TLR10-I775L, UBBP4-R2,
DISC1-R264 or FAM208A-I1374 had a statistically increased risk of 5-year OS (Table V).

We then included these parameters in a multivariate Cox regression analysis to clarify independent prognostic factors 
Table IV. The association between somatic mutations, 5-year DFS and 5-year OS ( $n=48)$.

\begin{tabular}{|c|c|c|c|c|c|c|c|c|c|c|}
\hline \multirow[t]{2}{*}{ Gene } & \multirow[t]{2}{*}{ SNP } & \multirow{2}{*}{$\begin{array}{l}\text { Mutation } \\
\text { site (a.a.) }\end{array}$} & \multicolumn{2}{|c|}{ Total cases $(\%)$} & \multicolumn{3}{|c|}{ 5-year DFS } & \multicolumn{3}{|c|}{ 5-year OS } \\
\hline & & & $\begin{array}{l}\text { WT } \\
(\%)\end{array}$ & $\begin{array}{c}\text { Variant } \\
(\%)\end{array}$ & $\begin{array}{c}\text { WT } \\
\text { survival } \\
(\%)\end{array}$ & $\begin{array}{c}\text { Variant } \\
\text { survival } \\
(\%)\end{array}$ & $p$-Value & $\begin{array}{c}\text { WT } \\
\text { survival } \\
(\%)\end{array}$ & $\begin{array}{c}\text { Variant } \\
\text { survival } \\
(\%)\end{array}$ & $p$-Value \\
\hline$R A S$ & & & $29(60.4 \%)$ & $19(39.6 \%)$ & $63.8 \%$ & $39.4 \%$ & 0.0533 & $74.5 \%$ & $45.0 \%$ & 0.0111 \\
\hline$R A S / B R A F$ & & & $27(56.3 \%)$ & $21(43.7 \%)$ & $69.1 \%$ & $35.4 \%$ & 0.0139 & $80.5 \%$ & $40.9 \%$ & 0.0012 \\
\hline$R A S / B R A F / P I K 3 C A$ & & & $26(54.2 \%)$ & $2245.8 \%)$ & $67.9 \%$ & $38.4 \%$ & 0.0308 & $79.8 \%$ & $43.6 \%$ & 0.0028 \\
\hline TIPIN & rs2063690 (c.332C>G) & A111G & $2858.3 \%)$ & $20(41.7 \%)$ & $61.9 \%$ & $44.3 \%$ & 0.171 & $76.8 \%$ & $45.3 \%$ & 0.0125 \\
\hline$T L R 1$ & rs5743618 (c.1805G >T) & S602I & $11(22.9 \%)$ & $37(77.1 \%)$ & $39.9 \%$ & $58.6 \%$ & 0.2436 & $62.0 \%$ & $64.4 \%$ & 0.8343 \\
\hline$T L R 1$ & $\mathrm{rs} 4833095(\mathrm{c} .743 \mathrm{~A}>\mathrm{G})$ & $\mathrm{N} 248 \mathrm{~S}$ & $3368.8 \%)$ & $15(31.2 \%)$ & $62.3 \%$ & $37.9 \%$ & 0.1377 & $75.1 \%$ & $40.7 \%$ & 0.025 \\
\hline$T T F 1$ & rs8999 $(\mathrm{c} .868 \mathrm{G}>\mathrm{T})$ & A290S & $21(43.8 \%)$ & $27(56.3 \%)$ & $64.3 \%$ & $46.3 \%$ & 0.1476 & $73.8 \%$ & $55.1 \%$ & 0.113 \\
\hline IGLV3-21 & rs4446155 (c.196G >A) & V66I & $12(25 \%)$ & $36(75 \%)$ & $73.0 \%$ & $48.3 \%$ & 0.1385 & $69.3 \%$ & $60.4 \%$ & 0.3462 \\
\hline$I G L V 3-21$ & $\mathrm{rs} 1985791(\mathrm{c} .202 \mathrm{G}>\mathrm{T})$ & D68Y & $11(22.9 \%)$ & $37(77.1 \%)$ & $70.3 \%$ & $49.7 \%$ & 0.2157 & $65.8 \%$ & $61.5 \%$ & 0.4754 \\
\hline IGSF3 & rs138851517 (c.1916T>C) & $\mathrm{I} 639 \mathrm{~T}$ & $45(93.8 \%)$ & $3(6.3 \%)$ & $58.5 \%$ & $0.0 \%$ & $<0.0001$ & $68.1 \%$ & $1.3 \%$ & $<0.0001$ \\
\hline$K I R 2 D S 4$ & rs4806590 (c.467A>G) & K156R & $37(77.1 \%)$ & $11(22.9 \%)$ & $60.6 \%$ & $34.6 \%$ & 0.129 & $66.9 \%$ & $53.7 \%$ & 0.3487 \\
\hline OR4D6 & rs1453541 (c.788T >C) & M263T & $31(64.6 \%)$ & $17(35.4 \%)$ & $48.7 \%$ & $64.3 \%$ & 0.2437 & $82.4 \%$ & $52.9 \%$ & 0.0274 \\
\hline OR6J1 & rs1753430 (c.748C >T) & $\mathrm{P} 250 \mathrm{~S}$ & $43(89.6 \%)$ & $5(10.4 \%)$ & $58.8 \%$ & $16.6 \%$ & 0.0138 & $66.4 \%$ & $36.4 \%$ & 0.0721 \\
\hline SNX31 & $\mathrm{rs} 2248609(\mathrm{c} .926 \mathrm{~A}>\mathrm{G})$ & E309R & $42(87.5 \%)$ & $6(12.5 \%)$ & $58.2 \%$ & $27.5 \%$ & 0.0989 & $68.7 \%$ & $27.8 \%$ & 0.0366 \\
\hline TLR10 & $\operatorname{rs} 4129009(\mathrm{c} .2323 \mathrm{~A}>\mathrm{G})$ & $\mathrm{I} 775 \mathrm{~L}$ & $32(66.7 \%)$ & $16(33.3 \%)$ & $63.8 \%$ & $36.3 \%$ & 0.0465 & $77.2 \%$ & $39.1 \%$ & 0.0059 \\
\hline$T L R 3$ & rs3775291 $(\mathrm{c} .1234 \mathrm{C}>\mathrm{T})$ & L412F & $26(54.2 \%)$ & $22(45.8 \%)$ & $46.6 \%$ & $63.3 \%$ & 0.2688 & $60.0 \%$ & $68.1 \%$ & 0.5618 \\
\hline TLR5 & rs5744174 (c.1846T>C) & F616L & $34(70.8 \%)$ & $14(29.2 \%)$ & $57.3 \%$ & $47.4 \%$ & 0.4681 & $69.9 \%$ & $48.9 \%$ & 0.1325 \\
\hline TLR8 & rs3764880 (c.1A>G) & M1V & $16(33.3 \%)$ & $32(66.7 \%)$ & $40.2 \%$ & $61.2 \%$ & 0.1106 & $54.6 \%$ & $67.6 \%$ & 0.239 \\
\hline TMPRSS11A & rs977728 (c.3G>A) & M1I & $41(85.4 \%)$ & $7(14.6 \%)$ & $86.5 \%$ & $47.4 \%$ & 0.0909 & $60.0 \%$ & $86.5 \%$ & 0.1935 \\
\hline UBBp4 & rs72842072 (c.5G>A) & $\mathrm{R} 2 \mathrm{E}$ & $46(95.8 \%)$ & $2(4.2 \%)$ & $57.2 \%$ & $1.4 \%$ & 0.0044 & $66.9 \%$ & $0.0 \%$ & 0.0003 \\
\hline FPRl & $\operatorname{rs} 867228(\mathrm{c} .1037 \mathrm{~A}>\mathrm{T})$ & E346A & $23(47.9 \%)$ & $25(52.1 \%)$ & $63.1 \%$ & $46.0 \%$ & 0.1676 & $72.0 \%$ & $55.1 \%$ & 0.1548 \\
\hline DISC1 & rs3738401 (c.791G>A) & R264E & $31(64.6 \%)$ & $17(35.4 \%)$ & $63.5 \%$ & $37.3 \%$ & 0.0568 & $74.0 \%$ & $44.5 \%$ & 0.0253 \\
\hline FAM208A & rs2291498 (c.4120A>G) & $\mathrm{I} 374 \mathrm{~V}$ & $45(93.8 \%)$ & $3(6.3 \%)$ & $58.3 \%$ & $3.6 \%$ & 0.0055 & $68.0 \%$ & $7.5 \%$ & 0.0004 \\
\hline
\end{tabular}

The survival outcomes were estimated by Kaplan-Meier survival analysis. RAS variants are defined as significant mutations in KRAS/NRAS gene. BRAF variant is defined as V600E mutation. PIK3CA variants is defined as significant mutations in PIK3CA gene.

for rectal adenocarcinoma (Table VI). Our results showed that the OR6J1-P250 variant $(\mathrm{HR}=5.638,95 \% \mathrm{CI}=1.379$ $19.816, p=0.0185)$ and IGSF3-I639 variant $(\mathrm{HR}=9.583,95 \%$ $\mathrm{CI}=1.286-81.499, p=0.0283)$ were independent prognostic factors for 5-year DFS, and the TIPIN-A111 (HR=6.356, 95\% CI=1.02-63.554, $p=0.0473)$, IGSF3-I639 $(\mathrm{HR}=74.518$, 95\% CI=4.434-2373.298, $p=0.0023), \quad$ OR4D6-M263 $(\mathrm{HR}=34.479,95 \% \mathrm{CI}=3.306-727.873, \quad p=0.0016)$ and DISC1-R264 $(\mathrm{HR}=5.622,95 \% \mathrm{CI}=1.171-35.822, p=0.0304)$ variants were independent prognostic factors for 5-year OS for rectal adenocarcinoma (Table VI). These data strongly indicate that these polymorphisms have significant clinical prognostic value for rectal adenocarcinoma.

\section{Discussion}

With the advance of NGS technologies, genetic variants have been gradually revealed to be associated with disease occurrence. Herein, we demonstrated the application of WES and a targeted sequence panel to identify the impact of germline genetic variants on clinical outcomes. Our results highlight that germline variants affect survival outcome and tumor relapse, which suggested that screening for the variants might be taken into consideration after treatment of rectal adenocarcinoma patients.

By comprehensive analysis of the targeted sequence panel and clinical database, we validated 9 germline variants that were significantly associated with 5-year DFS and 5-year OS. Several genetic variants were associated with cancer susceptibility and have been extensively reported. For example, TLR1-N248S (rs4833095) is associated with an increased risk of gastric cancer in a European population (27), IGSF3 variants are associated with an increased risk of hepatocarcinoma, ovarian cancer and colorectal cancer (28) and the TLR10-I775 (rs4129009) variant is associated with bladder cancer (29). Moreover, our study showed that several variants that have been implicated in disease occurrence are correlated with survival outcome in rectal adenocarcinoma. These results suggest that variants such as TIPIN-A111G (rs2063690), OR4D6-M263T (rs1453541), DISC1-I639T (rs3738401) and FAM208A-I1374V (rs2291498) might be cancer-related variants. Furthermore, we identified two novel 
Table V. Univariate analysis of somatic mutations, 5-year DFS and 5-year OS (n=48).

\begin{tabular}{|c|c|c|c|c|c|c|}
\hline \multirow[t]{2}{*}{ Gene } & \multicolumn{3}{|c|}{ 5-year DFS } & \multicolumn{3}{|c|}{ 5-year OS } \\
\hline & HR & $95 \% \mathrm{CI}$ & $p$-Value & HR & $95 \% \mathrm{CI}$ & $p$-Value \\
\hline Gender (Male vs. Female) & 1.169 & $0.475-3.282$ & 0.744 & 1.204 & $0.446-3.789$ & 0.7237 \\
\hline Age $(>65 v s .<65)$ & 1.569 & $0.639-3.714$ & 0.3153 & 1.796 & $0.673-4.71$ & 0.2351 \\
\hline cN stage (Positive vs. Negative) & 1.767 & $0.747-4.333$ & 0.1945 & 2.581 & $0.980-7.501$ & 0.055 \\
\hline pN stage (Positive $v s$. Negative) & 2.04 & $0.858-5.163$ & 0.1071 & 2.405 & $0.913-6.994$ & 0.0762 \\
\hline LVI (Present $v s$. Absent) & 3.339 & $1.349-9.407$ & 0.0085 & 3.145 & $1.161-9.921$ & 0.0237 \\
\hline PNI (Present vs. Absent) & 3.113 & $1.313-7.65$ & 0.0103 & 7.943 & $2.792-28.327$ & $<0.0001$ \\
\hline RAS (Variant $v s$. WT) & 2.278 & $0.062-05.476$ & 0.062 & 3.285 & $1.255-9.091$ & 0.0157 \\
\hline RAS/BRAF (Variants vs. WT) & 2.887 & $1.213-7.312$ & 0.0166 & 4.833 & $1.777-15.294$ & 0.0018 \\
\hline TIPIN (Variant $v s$. WT) & 1.805 & $0.76-4.337$ & 0.1781 & 3.311 & $1.258-9.627$ & 0.0152 \\
\hline TLR1-S602 (Variant vs. WT) & 0.572 & $0.231-1.61$ & 0.271 & 0.887 & $0.314-3.153$ & 0.8362 \\
\hline TLR1-N248 (Variant vs. WT) & 1.907 & $0.777-4.517$ & 0.1538 & 2.84 & $1.084-7.578$ & 0.0341 \\
\hline TTF1 (Variant $v s . \mathrm{WT}$ ) & 1.933 & $0.803-5.106$ & 0.1434 & 2.273 & $0.842-7.149$ & 0.107 \\
\hline IGLV3-21-V66(Variant vs. WT) & 2.446 & $0.827-10.447$ & 0.1128 & 1.806 & $0.589-7.837$ & 0.3239 \\
\hline IGLV3-21-D68 (Variant vs. WT) & 2.127 & $0.719-9.082$ & 0.1875 & 1.569 & $0.512-6.844$ & 0.4583 \\
\hline IGSF3 (Variant vs. WT) & 11.558 & $2.481-41.002$ & 0.0041 & 13.012 & $2.741-48.146$ & 0.0031 \\
\hline KIR2DS4 (Variant vs. WT) & 1.996 & $0.755-4.808$ & 0.1547 & 1.643 & $0.52-4.459$ & 0.3711 \\
\hline OR4D6 (Variant vs. WT) & 0.5723 & $0.204-1.412$ & 0.2334 & 0.267 & $0.061-0.827$ & 0.0202 \\
\hline OR6J1 (Variant vs. WT) & 3.638 & $1.038-9.951$ & 0.0443 & 2.991 & $0.675-9.239$ & 0.1286 \\
\hline SNX31 (Variant vs. WT) & 2.443 & $0.699-6.656$ & 0.1459 & 3.139 & $0.877-8.972$ & 0.0748 \\
\hline TLR10 (Variant $v s$. WT) & 2.332 & $0.97-5.544$ & 0.0582 & 3.569 & $1.369-9.845$ & 0.0096 \\
\hline TLR3 (Variant vs. WT) & 0.61 & $0.241-1.453$ & 0.2663 & 0.752 & $0.272-1.96$ & 0.5605 \\
\hline TLR5 (Variant vs. WT) & 1.398 & $0.529-3.362$ & 0.4791 & 2.069 & $0.75-5.4$ & 0.1534 \\
\hline TLR8 (Variant $v s . \mathrm{WT}$ ) & 0.5 & $0.211-1.229$ & 0.127 & 0.564 & $0.216-1.554$ & 0.2562 \\
\hline TMPRSS11A (Variant vs. WT) & 0.208 & $0.012-1.002$ & 0.0504 & 0.284 & $0.015-1.397$ & 0.1407 \\
\hline UBBP4 (Variant vs. WT) & 6.767 & $1.034-25.919$ & 0.0468 & 10.825 & $1.571-47.752$ & 0.0205 \\
\hline FPR1 (Variant $v s . \mathrm{WT})$ & 1.843 & $0.776-4.664$ & 0.167 & 2.031 & $0.771-5.905$ & 0.153 \\
\hline DISC1 (Variant $v s$. WT) & 2.263 & $0.937-5.402$ & 0.0687 & 2.852 & $1.083-7.643$ & 0.0342 \\
\hline FAM208A (Variant $v s$. WT) & 4.949 & $1.136-15.276$ & 0.0356 & 7.758 & $1.694-26.97$ & 0.012 \\
\hline
\end{tabular}

The log-rank tests were performed for univariate comparison. RAS variants are defined as significant mutations in KRAS/NRAS gene. BRAF variant is defined as V600E mutation.

Table VI. Multivariate analysis of somatic mutations in 5-year DFS and 5-year OS ( $\mathrm{n}=48)$.

\begin{tabular}{|c|c|c|c|c|c|c|}
\hline \multirow[t]{2}{*}{ Gene } & \multicolumn{3}{|c|}{ 5-year DFS } & \multicolumn{3}{|c|}{ 5-year OS } \\
\hline & HR & $95 \% \mathrm{CI}$ & $p$-Value & HR & $95 \% \mathrm{CI}$ & $p$-Value \\
\hline LVI (Present vs. Absent) & 1.829 & $0.593-5.891$ & 0.292 & 1.666 & $0.218-13.009$ & 0.618 \\
\hline PNI (Present vs. Absent) & 2.346 & $0.763-6.902$ & 0.1329 & 56.594 & $6.788-738.939$ & $<0.0001$ \\
\hline RAS/BRAF (Variants vs. WT) & 4.322 & $1.685-12.054$ & 0.0022 & 3.217 & $0.377-39.963$ & 0.3084 \\
\hline TIPIN-A111 (Variant $v s$. WT) & - & - & - & 6.356 & $1.02-63.554$ & 0.0473 \\
\hline TLR1-N248 (Variant vs. WT) & - & - & - & 3.472 & $0.755-19.063$ & 0.1105 \\
\hline IGSF3-I639 (Variant vs. WT) & 9.583 & $1.286-81.499$ & 0.0283 & 74.517 & $4.434-2373.298$ & 0.0023 \\
\hline OR4D6-M263 (Variant vs. WT) & - & - & - & 34.479 & $3.306-727.873$ & 0.0016 \\
\hline UBBP4-R2 (Variant vs. WT) & 3.124 & $0.223-24.489$ & 0.3638 & 0.129 & $0.003-3.317$ & 0.2216 \\
\hline FAM208A-I374 (Variant vs. WT) & 1.165 & $0.16-5.821$ & 0.8641 & 0.504 & $0.037-4.85$ & 0.5639 \\
\hline OR6J1-P250 (Variant vs. WT) & 5.638 & $1.379-19.816$ & 0.0185 & - & - & - \\
\hline TLR10-I775L (Variant vs. WT) & - & - & - & 1.412 & $0.149-11.915$ & 0.7515 \\
\hline DISC1-R264 (Variant vs. WT) & - & - & - & 5.622 & $1.171-35.822$ & 0.0304 \\
\hline
\end{tabular}

The Cox regression tests were performed for multivariate comparison. RAS variants are defined as significant mutations in K-RAS/N-RAS gene. BRAF variant is defined as V600E mutation. 
variants, SNX31-E309R (rs2248609) and UBBp4-R2E (rs72842072), that were correlated with poor 5-year DFS and 5-year OS. Taken together, our results suggest that these genetic variants are potential risk factors for tumor relapse and poor survival outcome in rectal adenocarcinoma. Importantly, by comparing to the pathogenic cancer-associated variants in the Clin Var database, we identified only two germline variants TLR1-N248S (rs4833095) and DISC1-I639T (rs3738401) have reported a relatively high frequency in diseases. These results indicated that patients with germline genetic variants, previously identified as non-oncogenic impacts should be carefully monitored. Therefore, precision oncology by NGS or targeted sequencing panel for germline variants seems to be a promising strategy for patients. But there are limitations in current study such as small sample size and different therapeutic strategies in rectal adenocarcinoma patient. More studies are needed to confirm the prognostic value of these variants in rectal adenocarcinoma.

Taken together, our findings highlight the importance of applying comprehensive genomic information to determine therapeutic strategies for rectal adenocarcinoma. Germline sequence results and tumor genomes have to be integratively analysed to provide more information on the overall genetic background of cancer development in rectal adenocarcinoma.

\section{Conflicts of Interest}

The Authors declare that no conflicts of interest exist.

\section{Authors' Contributions}

Kevin Chih-Yang Huang and Shu-Fen Chiang conducted and performed the experiments. William Tzu-Liang Chen, Tao-Wei Ke and Tsung-Wei Chen participated in the clinical information collection for rectal carcinoma patients. Shu-Fen Chiang performed the statistical analysis. Shu-Fen Chiang, William Tzu-Liang Chen and K. S. Clifford Chao supervised this study. Kevin Chih-Yang Huang, Shu-Fen Chiang and K. S. Clifford Chao analysed the data and wrote the manuscript.

\section{Acknowledgements}

This study was supported in part by China Medical University Hospital (DMR-108-099), Ministry of Science and Technology (MOST1072314-B-039-027-MY3, MOST107-2314-B-039-057-MY3 and MOST108-2320-B-039-045, Taiwan), Health and Welfare Surcharge Of Tobacco Products, and China Medical University Hospital Cancer Research Center of Excellence (MOHW108-TDU-B-212-124024, Taiwan). This study was partially based on clinical information from the China Medical University Hospital Cancer Registry.

\section{References}

1 Siegel RL, Miller KD and Jemal A: Cancer statistics, 2017. CA Cancer J Clin 67(1): 7-30, 2017. PMID: 28055103. DOI: $10.3322 /$ caac. 21387
2 Conde-Muino R, Cuadros M, Zambudio N, Segura-Jimenez I, Cano $\mathrm{C}$ and Palma P: Predictive biomarkers to chemoradiation in locally advanced rectal cancer. Biomed Res Int 2015: 921435 , 2015. PMID: 26504848. DOI: 10.1155/2015/921435

3 Sauer R, Liersch T, Merkel S, Fietkau R, Hohenberger W, Hess C, Becker H, Raab HR, Villanueva MT, Witzigmann H, Wittekind $\mathrm{C}$, Beissbarth T and Rodel C: Preoperative versus postoperative chemoradiotherapy for locally advanced rectal cancer: Results of the german $\mathrm{CAO} / \mathrm{ARO} / \mathrm{AIO}-94$ randomized phase III trial after a median follow-up of 11 years. J Clin Oncol 30(16): 1926-1933, 2012. PMID: 22529255. DOI: 10.1200/JCO.2011.40.1836

4 Yoon WH, Kim HJ, Kim CH, Joo JK, Kim YJ and Kim HR: Oncologic impact of pathologic response on clinical outcome after preoperative chemoradiotherapy in locally advanced rectal cancer. Ann Surg Treat Res 88(1): 15-20, 2015. PMID: 25553320. DOI: 10.4174/astr.2015.88.1.15

5 Takeda M, Kawahara H, Ogawa M, Suwa K, Eto KEN and Yanaga K: Reevaluation of preoperative chemoradiotherapy for clinical T3 lower rectal cancer: A multicenter collaborative retrospective clinical study. Anticancer Res 39(6): 3047-3052, 2019. PMID: 31177147 . DOI: 10.21873 /anticanres.13438

6 Balko JM and Black EP: A gene expression predictor of response to EGFR-targeted therapy stratifies progression-free survival to cetuximab in KRAS wild-type metastatic colorectal cancer. BMC Cancer 9: 145, 2009. PMID: 19439077. DOI: 10.1186/1471-2407-9-145

7 Babaei K, Khaksar R, Zeinali T, Hemmati H, Bandegi A, Samidoust P, Ashoobi MT, Hashemian H, Delpasand K, Talebinasab F, Naebi H, Mirpour SH, Keymoradzadeh A and Norollahi SE: Epigenetic profiling of MUTYH, KLF6, WNT1 and KLF4 genes in carcinogenesis and tumorigenesis of colorectal cancer. BioMedicine 9(4), 2019. PMID: 31724937. DOI: $10.1051 / \mathrm{bmdcn} / 2019090422$

8 Alexandrov LB, Nik-Zainal S, Siu HC, Leung SY and Stratton MR: A mutational signature in gastric cancer suggests therapeutic strategies. Nat Commun 6: 8683, 2015. PMID: 26511885. DOI: $10.1038 /$ ncomms 9683

9 Sabattini E, Bacci F, Sagramoso C and Pileri SA: Who classification of tumours of haematopoietic and lymphoid tissues in 2008: An overview. Pathologica 102(3): 83-87, 2010. PMID: 21171509.

10 Rotow $\mathrm{J}$ and Bivona TG: Understanding and targeting resistance mechanisms in NSCLC. Nat Rev Cancer 17(11): 637-658, 2017. PMID: 29068003. DOI: 10.1038/nrc.2017.84

11 Zhang J, Zheng J, Yang Y, Lu J, Gao J, Lu T, Sun J, Jiang H, Zhu Y, Zheng Y, Liang Z and Liu T: Molecular spectrum of KRAS, NRAS, BRAF and PIK3CA mutations in chinese colorectal cancer patients: Analysis of 1,110 cases. Sci Rep 5: 18678, 2015. PMID: 26691448. DOI: 10.1038/srep18678

12 Zehir A, Benayed R, Shah RH, Syed A, Middha S, Kim HR, Srinivasan P, Gao J, Chakravarty D, Devlin SM, Hellmann MD, Barron DA, Schram AM, Hameed M, Dogan S, Ross DS, Hechtman JF, DeLair DF, Yao J, Mandelker DL, Cheng DT, Chandramohan R, Mohanty AS, Ptashkin RN, Jayakumaran G, Prasad M, Syed MH, Rema AB, Liu ZY, Nafa K, Borsu L, Sadowska J, Casanova J, Bacares R, Kiecka IJ, Razumova A, Son JB, Stewart L, Baldi T, Mullaney KA, Al-Ahmadie H, Vakiani E, Abeshouse AA, Penson AV, Jonsson P, Camacho N, Chang MT, Won HH, Gross BE, Kundra R, Heins ZJ, Chen HW, Phillips S, Zhang H, Wang J, Ochoa A, Wills J, Eubank M, 
Thomas SB, Gardos SM, Reales DN, Galle J, Durany R, Cambria R, Abida W, Cercek A, Feldman DR, Gounder MM, Hakimi AA, Harding JJ, Iyer G, Janjigian YY, Jordan EJ, Kelly CM, Lowery MA, Morris LGT, Omuro AM, Raj N, Razavi P, Shoushtari AN, Shukla N, Soumerai TE, Varghese AM, Yaeger R, Coleman J, Bochner B, Riely GJ, Saltz LB, Scher HI, Sabbatini PJ, Robson ME, Klimstra DS, Taylor BS, Baselga J, Schultz N, Hyman DM, Arcila ME, Solit DB, Ladanyi M and Berger MF: Mutational landscape of metastatic cancer revealed from prospective clinical sequencing of 10,000 patients. Nat Med 23(6): 703-713, 2017. PMID: 28777785. DOI: $10.1038 / \mathrm{nm} .4333$

13 Lin PC, Yeh YM, Wu PY, Hsu KF, Chang JY and Shen MR: Germline susceptibility variants impact clinical outcome and therapeutic strategies for stage iii colorectal cancer. Sci Rep 9(1): 3931, 2019. PMID: 30850667. DOI: 10.1038/s41598-01940571-0

14 Margetis N, Kouloukoussa M, Pavlou K, Vrakas S and MariolisSapsakos T: K-ras mutations as the earliest driving force in a subset of colorectal carcinomas. In Vivo 31(4): 527-542, 2017. PMID: 28652417. DOI: 10.21873/invivo.11091

15 Pikoulis E, Margonis GA, Andreatos N, Sasaki K, Angelou A, Polychronidis G, Pikouli A, Riza E, Pawlik TM and Antoniou E: Prognostic role of BRAF mutations in colorectal cancer liver metastases. Anticancer Res 36(9): 4805-4812, 2016. PMID: 27630332. DOI: 10.21873 /anticanres. 11040

16 Huang CY, Chiang SF, Ke TW, Chen TW, You YS, Chen WTL and Chao KSC: Clinical significance of programmed death 1 ligand-1 (CD274/PD-L1) and intra-tumoral CD8+T-cell infiltration in stage II-III colorectal cancer. Sci Rep 8(1): 15658, 2018. PMID: 30353144. DOI: 10.1038/s41598-018-33927-5

17 Lièvre A, Bachet J-B, Le Corre D, Boige V, Landi B, Emile JF, Côté J-F, Tomasic G, Penna C, Ducreux M, Rougier P, Penault-Llorca $\mathrm{F}$ and Laurent-Puig P: KRAS mutation status is predictive of response to cetuximab therapy in colorectal cancer. Cancer Res 66(8): 3992-3995, 2006. PMID: 16618717. DOI: 10.1158/0008-5472.Can-06-0191

18 Hsu HC, Thiam TK, Lu YJ, Yeh CY, Tsai WS, You JF, Hung HY, Tsai CN, Hsu A, Chen HC, Chen SJ and Yang TS: Mutations of KRAS/NRAS/BRAF predict cetuximab resistance in metastatic colorectal cancer patients. Oncotarget 7(16): 22257-22270, 2016. PMID: 26989027. DOI: 10.18632/oncotarget.8076

19 Teutsch SM, Bradley LA, Palomaki GE, Haddow JE, Piper M, Calonge N, Dotson WD, Douglas MP, Berg AO and Group EW: The evaluation of genomic applications in practice and prevention (EGAPP) initiative: Methods of the egapp working group. Genet Med 11(1): 3-14, 2009. PMID: 18813139. DOI: 10.1097/GIM.0b013e318184137c

20 Huang CY, Chiang SF, Chen WTL, Ke TW, Chen TW, You YS, Lin CY and Chao KSC: HMGB1 promotes ERK-mediated mitochondrial DRP1 phosphorylation for chemoresistance through rage in colorectal cancer. Cell Death Dis 9(10): 1004, 2018. PMID: 30258050. DOI: 10.1038/s41419-018-1019-6

21 Chen TW, Huang KCY, Chiang SF, Chen WTL, Ke TW and Chao KSC: Prognostic relevance of programmed cell deathligand 1 expression and CD8+TILs in rectal cancer patients before and after neoadjuvant chemoradiotherapy. J Cancer Res Clin Oncol 145(4): 1043-1053, 2019. PMID: 30874889. DOI: $10.1007 / \mathrm{s} 00432-019-02874-7$
22 Di J, Yang H, Jiang B, Wang Z, Ji J and Su X: Whole exome sequencing reveals intertumor heterogeneity and distinct genetic origins of sporadic synchronous colorectal cancer. Int J Cancer. 142(5): 927-939, 2017. PMID: 29105743. DOI: 10.1002/ ijc. 31140

23 Huang CY, Chiang SF, Ke TW, Chen TW, Lan YC, You YS, Shiau AC, Chen WT and Chao KSC: Cytosolic high-mobility group box protein 1 (HMGB1) and/or PD-1+ TILs in the tumor microenvironment may be contributing prognostic biomarkers for patients with locally advanced rectal cancer who have undergone neoadjuvant chemoradiotherapy. Cancer Immunol Immunother 67(4): 551-562, 2018. PMID: 29270668. DOI: 10.1007/s00262-017-2109-5

24 Wang X, Sheu JJ, Lai MT, Yin-Yi Chang C, Sheng X, Wei L, Gao Y, Wang X, Liu N, Xie W, Chen CM, Ding WY and Sun L: RSF-1 overexpression determines cancer progression and drug resistance in cervical cancer. BioMedicine $8(1)$ : 4, 2018. PMID: 29480799. DOI: 10.1051/bmdcn/2018080104

25 Chiang SF, Huang CY, Ke TW, Chen TW, Lan YC, You YS, Chen WTL and Chao KSC: Upregulation of tumor PD-L1 by neoadjuvant chemoradiotherapy (neoCRT) confers improved survival in patients with lymph node metastasis of locally advanced rectal cancers. Cancer Immunol Immunother 68(2): 283-296, 2019. PMID: 30448924. DOI: 10.1007/s00262-0182275-0

26 Cho HJ, Baek JH, Baek DW, Kang BW, Lee SJ, Kim HJ, Park SY, Park JS, Choi GS and Kim JG: Prognostic significance of clinicopathological and molecular features after neoadjuvant chemoradiotherapy in rectal cancer patients. In Vivo 33(6): 19591965, 2019. PMID: 31662525. DOI: 10.21873/invivo.11691

27 Dargiene G, Streleckiene G, Skieceviciene J, Leja M, Link A, Wex T, Kupcinskas L, Malfertheiner P and Kupcinskas J: TLR1 and PRKAA1 gene polymorphisms in the development of atrophic gastritis and gastric cancer. J Gastrointestin Liver Dis 27(4): 363 369, 2018. PMID: 30574617. DOI: 10.15403/jgld.2014.1121.274.tlr

28 DeRycke MS, Gunawardena SR, Middha S, Asmann YW, Schaid DJ, McDonnell SK, Riska SM, Eckloff BW, Cunningham JM, Fridley BL, Serie DJ, Bamlet WR, Cicek MS, Jenkins MA, Duggan DJ, Buchanan D, Clendenning M, Haile RW, Woods MO, Gallinger SN, Casey G, Potter JD, Newcomb PA, Le Marchand L, Lindor NM, Thibodeau SN and Goode EL: Identification of novel variants in colorectal cancer families by high-throughput exome sequencing. Cancer Epidemiol Biomarkers Prev 22(7): 1239-1251, 2013. PMID: 23637064. DOI: $10.1158 / 1055-9965 . E p i-12-1226$

29 Guirado M, Gil H, Saenz-Lopez P, Reinboth J, Garrido F, Cozar JM, Ruiz-Cabello F and Carretero R: Association between C13ORF31, NOD2, RIPK2 and TLR10 polymorphisms and urothelial bladder cancer. Hum Immunol 73(6): 668-672, 2012. PMID: 22504414. DOI: 10.1016/j.humimm.2012.03.006
Received February 1, 2020

Revised February 21, 2020

Accepted February 29, 2020 